\title{
Feasibility studies for assaying alpha-fetoprotein using antibody-activated magnetic nanoparticles
}

This article was published in the following Dove Press journal:

International Journal of Nanomedicine

16 April 2012

Number of times this article has been viewed

\section{Kai-Wen Huang' \\ Shieh-Yueh Yang ${ }^{2,3}$ \\ Yu-Wei Hong ${ }^{3}$ \\ Jen-Jie Chieh ${ }^{3}$ \\ Che-Chuan Yang ${ }^{3}$ \\ Herng-Er Horng ${ }^{3}$ \\ Chau-Chung $\mathrm{Wu}^{4}$ \\ Chin-Yih Hong 5 \\ Hong-Chang Yang ${ }^{6}$ \\ 'Department of Surgery and \\ Hepatitis Research Center, National \\ Taiwan University Hospital, National \\ Taiwan University, Taipei, ${ }^{2} \mathrm{MagQu}$ \\ Co, Ltd, Sindian Dist, New Taipei \\ City, ${ }^{3}$ Institute of Electro-optical \\ Science and Technology, National \\ Taiwan Normal University, Taipei, \\ ${ }^{4}$ Departments of Internal Medicine \\ and Primary Care Medicine, College \\ of Medicine, National Taiwan \\ University, Taipei, ${ }^{5} \mathrm{Graduate}$ Institute \\ of Bio-medical Engineering, National \\ Chung Hsing University, Taichung, \\ ${ }^{6}$ Department of Physics, National \\ Taiwan University, Taipei, Taiwan}

Correspondence: Herng-Er Horng Institute of Electro-optical Science and

Technology, National Taiwan Normal

University, Taipei II6,

Taiwan, Republic of China

Tel +8862 7734 6712

Fax +886286631954

Email phyfv00I@ntnu.edu.tw

Chau-Chung Wu

Departments of Internal Medicine

and Primary Care Medicine,

College of Medicine, National Taiwan

University, Taipei 100, Taiwan, Republic of China

Tel +886 223123456

Fax +886 2239| 4853

Email chauchungwu@ntu.edu.tw

\begin{abstract}
Some previous reports have already shown the characterizations of immunomagnetic reduction (IMR). The assay technology involves the utilities of biofunctionalized magnetic nanoparticles to label target biomolecules. However, the detection threshold and interference tests for IMR have not been investigated in detail. In this study, alpha-fetoprotein (AFP) was used as a target biomolecule. The signals for AFP solutions of various concentrations, or with interfering materials, were detected via IMR. These samples were also used for characterizing the detection threshold and interference with enzyme-linked immunosorbent assay (ELISA). The results of assaying AFP level with IMR and ELISA were compared. The detection threshold for assaying AFP with IMR was found to be $3 \mathrm{ng} / \mathrm{mL}$, which is 15 times lower than that of ELISA, and definitely suppresses false negative. For the interfering materials noted commonly in serum such as hemoglobin, bilirubin, triglyceride, and vascular endothelial growth factor, there was no detectable interfering effect when assaying AFP with IMR. Several serum samples from normal people and liver-tumor-bearing patients were used for the detections of AFP concentration via IMR. These results reveal the feasibilities of assaying AFP in blood using IMR, as well as achieving high-sensitive and high-specific assay for AFP.
\end{abstract}

Keywords: immunomagnetic reduction, ELISA, biofunctionalized magnetic nanoparticles

\section{Introduction}

To improve the sensitivity and specificity of immunoassay, the developing trends are to lower the detection threshold and to minimize the cross reaction. Many research groups have improved in-use assay technologies. ${ }^{1-3}$ Some other groups have explored advanced technologies. ${ }^{4-7}$

Several years ago, some authors proposed an assay technology called immunomagnetic reduction (IMR). ${ }^{5}$ In IMR, the reagent is a solution of homogeneously dispersed magnetic nanoparticles, which are coated with hydrophilic surfactants (eg, dextran) and bioprobes (eg, antibodies). Under external multiple alternating-current (ac) magnetic fields, magnetic nanoparticles oscillate with the multiple ac magnetic fields via magnetic interaction (Figure 1A). Thus, the reagents under the external multiple ac magnetic fields show a magnetic property, called mixed-frequency ac magnetic susceptibility $\chi_{\mathrm{ac}}$. With the bioprobes on the outmost shell, magnetic nanoparticles bind with and magnetically label the biomolecules (eg, target biomolecules) to be detected. Due to the binding, magnetic nanoparticles become larger or clustered (Figure 1B). The responses of these larger or clustered magnetic nanoparticles to external multiple ac magnetic fields are much less intense than those of the original individual magnetic nanoparticles. Thus, the $\chi_{\text {ac }}$ of the reagent is reduced due to the binding between the 
A $\chi_{\mathrm{ac}}, 0$

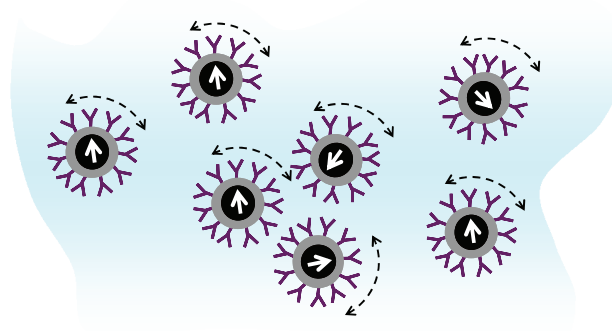

B $\chi_{\mathrm{ac}, \phi}\left(<\chi_{\mathrm{ac}, 0}\right)$

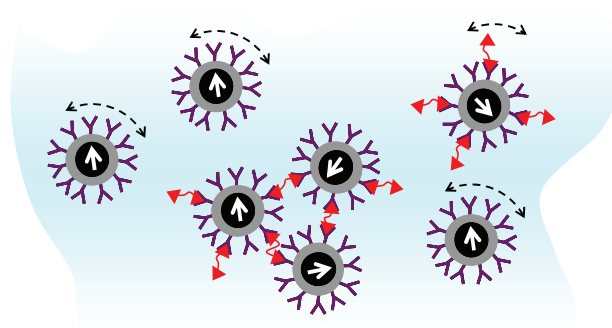

Figure I Illustration of mechanism of immunomagnetic reduction to detect biotargets. (A) Each magnetic nanoparticle oscillates individually with the applied alternative-current magnetic field before binding with biotargets. (B) Portions of magnetic nanoparticles become larger due to the binding with biotargets. The bound magnetic nanoparticles in (B) contribute to the reduction in the alternative-current magnetic susceptibility of the reagent.

magnetic nanoparticles and target biomolecules. This is why the technology is referred to as immunomagnetic reduction. In principle, when greater amounts of to-be-detected biomolecules are mixed with a reagent, more magnetic nanoparticles become larger. Thus, a larger reduction in $\chi_{\mathrm{ac}}$ could be expected for the reagents.

To quantify the reduction in the $\chi_{\mathrm{ac}}$ due to the binding between magnetic nanoparticles and biomolecules, this report refers to $\chi_{\mathrm{ac}}$ as $\chi_{\mathrm{ac}, 0}$ for the magnetic reagent without binding with the detected biomolecules, and as $\chi_{\mathrm{ac}, \phi}$ for the magnetic reagent after the binding between magnetic nanoparticles and biomolecules. The reduction in $\chi_{\mathrm{ac}}$ hereafter is defined as:

$$
\operatorname{IMR}(\%)=\left(\chi_{\mathrm{ac}, \mathrm{o}}-\chi_{\mathrm{ac}, \phi}\right) / \chi_{\mathrm{ac}, \phi} \times 100 \%
$$

According to the description given above, IMR exhibits several unique merits. Firstly, it is not necessary to remove the unbound target biomolecules and magnetic nanoparticles. They are still in the reagent. Therefore, the assay process of IMR is simple. Secondly, only one kind of bioprobe is used. Thirdly, IMR is a direct and homogeneous assay, which usually shows high reliability. Fourth, because the amount of reduction in $\chi_{\mathrm{ac}}$ can be accurately measured to correspond to the concentration of the to-be-detected biomolecules, the concentration of the biomolecules can thus be measured quantitatively.

Several papers have demonstrated that IMR can be applied to assay proteins, ${ }^{8}$ viruses, ${ }^{9}$ chemicals, and nucleic $\operatorname{acids}^{10}$ once suitable bioprobes are immobilized onto the magnetic nanoparticles. However, investigations of the detection threshold and interference tests of IMR have been very rare. Moreover, few studies have made comparisons between IMR and in-use assays. These uncertainties make it difficult to evaluate the accuracy or feasibilities of using IMR for clinic applications.

This study examined the detection threshold and interference tests for IMR. Alpha-fetoprotein (AFP) was selected as the target biomolecule. To determine the detection threshold for assaying AFP, the relationship between the AFP concentration and IMR (\%) was built up experimentally. As to the interference tests, the contributions from the commonly existing biomolecules in human blood to IMR (\%) were clarified. All these results were compared with those obtained with certificated assays in current practice, such as enzyme-linked immunosorbent assay (ELISA).

\section{Materials and methods}

The reagent for assaying AFP consists of magnetic nanoparticles (MF-DEX-0060, MagQu, New Taipei, Taiwan, Republic of China) functionalized with antibodies (ab40942; Abcam, Cambridge, MA), against AFP (EA502-Q1053; EastCoast Bio, North Berwick, ME). ${ }^{11}$ The mean diameter of the antibody-functionalized magnetic nanoparticles is $57.3 \mathrm{~nm}$. These magnetic nanoparticles are dispersed in phosphoryl buffer solution (PBS). The concentration of the magnetic bioreagent is $1.2 \mathrm{mg}-\mathrm{Fe} / \mathrm{mL}$.

$40-\mu \mathrm{L}$ of magnetic bioreagent was mixed with $60 \mu \mathrm{L}$ of sample solution in a glass tube. The mixed-frequency ac magnetic susceptibility $\chi_{\mathrm{ac}}$ of the mixture was detected as a function of time by using an IMR analyzer (XacPro-E; $\mathrm{MagQu}$ ). The reduction percentage in $\chi_{\mathrm{ac}}$, denoted as IMR (\%), can be determined according to the time dependent $\chi_{\mathrm{ac}}$ of the mixture.

The commercial ELISA kit (AF064T; Calbiotech, Spring Valley, CA) for assaying AFP was used in this work. The optical density at the wavelength of $450 \mathrm{~nm}\left(\mathrm{OD}_{450}\right)$ was detected using an ELISA reader (Plus ${ }^{384}$; Spectra Max, Sunnyvale, CA). The procedures used for AFP assay followed the protocols suggested by the commercial kits.

Various amounts of AFP were spiked into PBS for IMR assay. The concentrations of AFP solution varied from 
Table I Information on interference materials used for the IMR and ELISA interference tests in this work

\begin{tabular}{llll}
\hline Interfering material & Related disease & Normal reference level & Concentration used \\
\hline Hemoglobin & Hemolysis & $<500 \mu \mathrm{g} / \mathrm{mL}$ & $600 \mu \mathrm{g} / \mathrm{mL}$ \\
Conjugated bilirubin & Jaundice & $<2 \mu \mathrm{g} / \mathrm{mL}$ & $5 \mu 00 \mu \mathrm{g} / \mathrm{mL}$ \\
& & & $5 \mathrm{~g} / \mathrm{mL}$ \\
Triglyceride & Hypertriglyceridemia & $500-1500 \mu \mathrm{g} / \mathrm{mL}$ & $10 \mu \mathrm{mL}$ \\
Vascular endothelial growth factor & Malignancy & $<0.05 \mathrm{ng} / \mathrm{mL}$ & $2000 \mu \mathrm{g} / \mathrm{mL}$ \\
\hline
\end{tabular}

Abbreviations: IMR, immunomagnetic reduction; ELISA, enzyme-linked immunosorbent assay.

$0.1 \mathrm{ng} / \mathrm{mL}$ to $2000 \mathrm{ng} / \mathrm{mL}$. These AFP solutions were used for establishing the AFP concentration-dependent IMR (\%).

For interference tests, materials including hemoglobin (Hb) (H7379-1G; Sigma-Aldrich, St Louis, MO), conjugated bilirubin (C-BL) (14370-250MG; Sigma), triglyceride (TG) (T2449-10ML, Sigma), and vascular endothelial growth factor (VEGF) (sc-7269; Santa Cruz Biotechnology, Santa Cruz, CA), were mixed with AFP solutions, respectively. The concentrations of the interference materials used here are listed in Table 1. The related diseases and normal concentration levels for these interference materials were also tabulated. The IMR (\%) for each sample with interference material was compared with that of pure AFP solution. For each sample, the IMR (\%) was detected in triplicate.

\section{Results and discussion}

The experimental relationship between the IMR (\%) and AFP concentration $\phi_{\text {AFP }}$ is shown with dots (Figure 2). The IMR (\%) varies from $1.07 \%$ to $2.51 \%$ as $\phi_{\mathrm{AFP}}$ increases from $0.1 \mathrm{ng} / \mathrm{mL}$ to $2000 \mathrm{ng} / \mathrm{mL}$. The dots in Figure 2 are fitted to the following logistic function:

$$
\operatorname{IMR}(\%)=\left[\frac{\mathrm{A}-\mathrm{B}}{1+\left(\frac{\phi_{\mathrm{AFP}}}{\phi_{\mathrm{o}}}\right)^{\gamma}}+\mathrm{B}\right](\%)
$$

where $\mathrm{A}, \mathrm{B}, \phi_{\mathrm{o}}$, and $\gamma$ are fitting parameters. These parameters are found as $\mathrm{A}=1.13, \mathrm{~B}=2.76, \phi_{\mathrm{o}}=197.5$, and $\gamma=0.78$. The fitting curve is plotted with the solid line (Figure 2). The coefficient of determination, $\mathrm{R}^{2}$, between the dots and the fitting curve is 0.994 .

These AFP solutions were also used for ELISA assay. The detected $\mathrm{OD}_{450}$ as a function of $\phi_{\mathrm{AFP}}$ is shown with hollow squares (Figure 2). These hollow squares are guided with a dashed line. It is obvious that $\mathrm{OD}_{450}$ increases significantly as $\phi_{\mathrm{AFP}}$ surpasses $50 \mathrm{ng} / \mathrm{mL}$.

According to the dots (Figure 2$)$, the IMR (\%)- $\phi_{\text {AFP }}$ curve follows the logistic function, as expressed in Equation (2). The fitting parameter A (=1.13) in Equation (2) denotes the noise level for the IMR (\%)- $\phi_{\mathrm{AFP}}$ curve. The detection threshold in terms of the detected signal is conventionally defined as that higher than the noise level with triple standard deviations for the detected signal at low concentrations. In this work, the standard deviation of IMR (\%) for $1 \mathrm{ng} / \mathrm{mL}$ is $0.02 \%$. The detection threshold in terms of IMR (\%) is $1.19 \%$. Thus, the detection threshold in terms of AFP concentration $\phi_{\mathrm{AFP}}$ can be determined via Equation (2), which results in $3.01 \mathrm{ng} / \mathrm{mL}$. Obviously, the detection threshold is lowered by 15 times when using IMR instead of ELISA. The improvement in the detection threshold for IMR compared with ELISA is attributed from several factors, such as antibodies, uniformity of magnetic particles, and the detecting modules for magnetic signals. From the assay system point of view, IMR definitely shows merits in terms of the detection threshold.

In practice, the reference criteria of the AFP serum level for diagnosis of hepatocellular carcinoma (HCC) are above $20 \mathrm{ng} / \mathrm{mL}$. According to the results (Figure 2), IMR shows the detection threshold of $3.01 \mathrm{ng} / \mathrm{mL}$, which is much lower

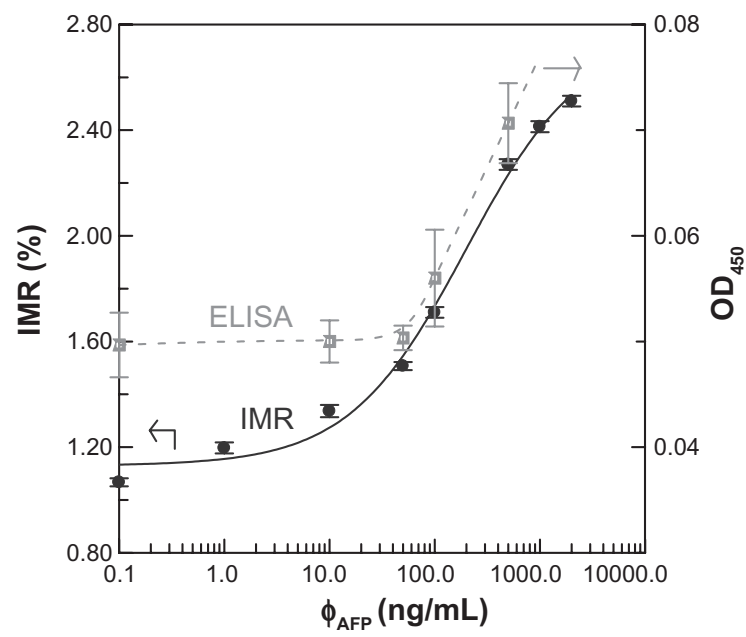

Figure 2 AFP concentration-dependent IMR (\%) (solid circles with the solid line) and $\mathrm{OD}_{450}$ (open squares with the dashed line) obtained with IMR and ELISA, respectively.

Note: Points represent mean \pm standard deviation.

Abbreviations: AFP, alphafetaprotein; IMR, immunomagnetic reduction; ELISA, enzyme-linked immunosorbent assay; OD, optical density. 
than $20 \mathrm{ng} / \mathrm{mL}$. This means that it is highly sensitive (or low false negative) for assaying AFP. To demonstrate this point, six serum samples of early-stage HCC patients were used for the AFP assay with both ELISA and IMR, respectively. The results are shown in Figure 3. It was found that the three samples showed AFP concentrations lower than $20 \mathrm{ng} / \mathrm{mL}$. This means that a high false negative exists. However, when using IMR, the detected AFP concentrations for these six serum samples were higher than $20 \mathrm{ng} / \mathrm{mL}$. Moreover, all the six samples were positive according to the IMR results. Thus, the ultra-low detection threshold for assaying AFP achieved by IMR definitely suppresses the false negative.

The IMR (\%) for AFP solutions without or with interfering materials were detected. The results are shown in Figure 4A. The AFP concentration in each sample is $500 \mathrm{ng} / \mathrm{mL}$. The pure AFP solution does not contain interfering material, and the label for interfering material is "None" for the pure AFP solution (Figure 4A). It is worth noting that all the samples exhibit IMR (\%) around $2.27 \%$. No definite deviations in IMR (\%) were found among pure AFP solutions and those with interfering materials. Therefore, regardless of what materials were used, $\mathrm{Hb}, \mathrm{C}-\mathrm{BL}$, TG, or VEGF, no detectable interference was shown for IMR assay on AFP.

The interference tests for AFP assay with ELISA were also investigated. The $\mathrm{OD}_{450}$ for each of the samples used in

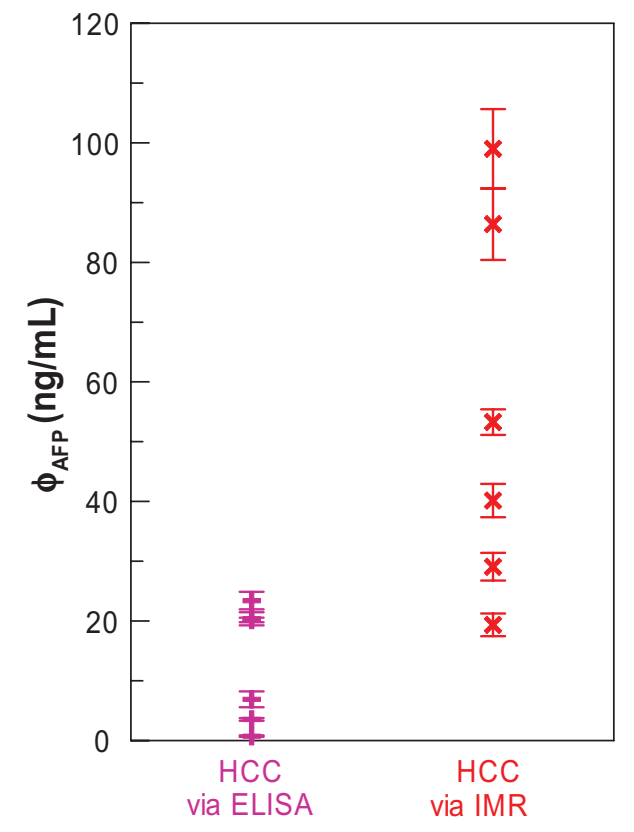

Figure 3 AFP concentration in sera of early-stage HCC patients detected by ELISA and IMR, respectively.

Note: Points represent mean \pm standard deviation.

Abbreviations: AFP, alphafetaprotein; HCC, hepatocellular carcinoma; ELISA, enzyme-linked immunosorbent assay; IMR, hepatocellular carcinoma.
Figure 4A are shown in Figure 4B. For the pure AFP solution labeled with "None" in Figure $4 \mathrm{~B}$, the $\mathrm{OD}_{450}$ is $0.07 \pm 0.003$. However, for the AFP solution with interfering materials, the signals of $\mathrm{OD}_{450}$ are higher than 0.45 . The results shown in Figure 4B reveal that materials such as $\mathrm{Hb}, \mathrm{C}-\mathrm{BL}, \mathrm{TG}$, and VEGF contribute a lot of interference to $\mathrm{OD}_{450}$ in the ELISA on AFP.

The nonsignificant interference from $\mathrm{Hb}, \mathrm{C}-\mathrm{BL}, \mathrm{TG}$, and VEGF for AFP assay with IMR is shown in Figure 4A. This evidence suggests that a high specificity could be achieved for assaying AFP with IMR. The interference for immunoassay is mainly attributed to sample color and cross reaction. The AFP solution with $\mathrm{Hb} / \mathrm{C}-\mathrm{BL} / \mathrm{TG}$ is faintly red/orange/white. The sample color affects the emission/ transmission/absorption of fluorescent markers in ELISA. In addition, non-specific binding exists between antibodies and nontarget molecules. Therefore, the $\mathrm{OD}_{450}$ of the
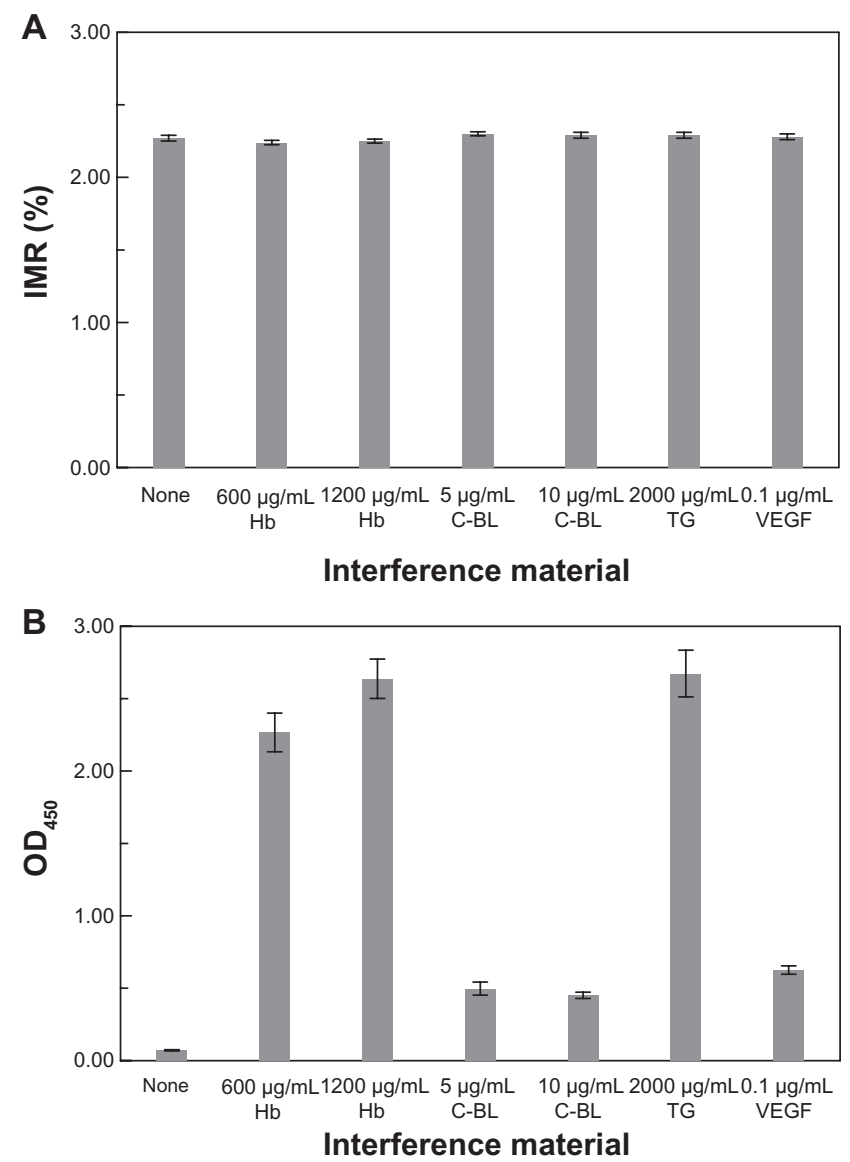

Figure 4 Contributions from various kinds of interfering materials to (A) IMR (\%) and (B) $O D_{450}$ measured with IMR and ELISA, respectively. The AFP concentration in each sample is $500 \mathrm{ng} / \mathrm{mL}$.

Notes: The label "None" means there is no interfering material in the $500-\mathrm{ng} / \mathrm{mL}$ AFP solution; bars represent mean \pm standard deviation.

Abbreviations: AFP, alphafetaprotein; IMR, immunomagnetic reduction; ELISA, enzyme-linked immunosorbent assay; OD, optical density; VEGF, vascular endothelial growth factor. 


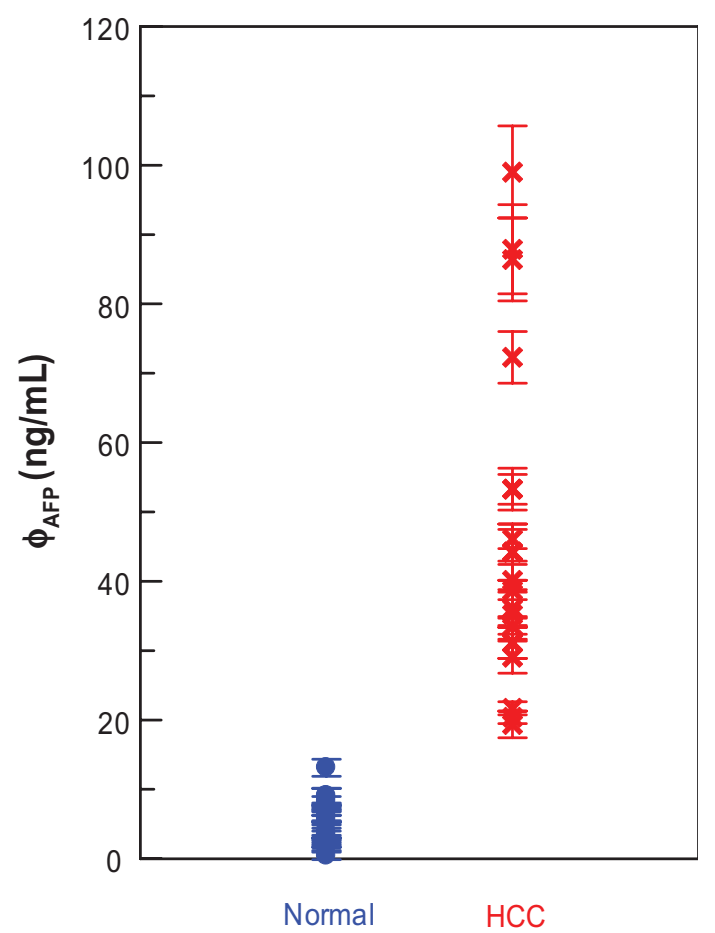

Figure 5 AFP concentrations in sera of normal people and HCC patients. The AFP concentrations are detected via IMR. The sample numbers are 17 for the normal group and 19 for the HCC group.

Note: Points represent mean \pm standard deviation.

Abbreviations: AFP, alphafetaprotein; HCC, hepatocellular carcinoma; IMR, immunomagnetic reduction.

AFP solution with interfering materials differs from that of the pure AFP solution. However, the signal detected with IMR is magnetic. The magnetic signals are transparent to any sample color. Hence, the sample color does not cause interference for IMR.

As for the nonspecific binding between the antibodies and nontarget molecules, it is significantly depressed with the centrifugal force acting on the nontarget molecules under ac magnetic fields. The detailed mechanism for the depression in the nonspecific binding for IMR has been clarified in a previous study. ${ }^{12}$ Briefly speaking, magnetic nanoparticles oscillate with the external ac magnetic fields when detecting the $\chi_{\mathrm{ac}}$ of the magnetic reagent. The nontarget molecules originally bound with antibodies on the oscillating magnetic nanoparticles experience centrifugal forces. At high oscillating frequencies, the centrifugal force is enhanced to break down the nonspecific binding. However, it is still weaker than the specific binding force between anti-AFP and AFP molecules. As a result, the cross reactions are inhibited for IMR.

Nineteen serum samples of patients with HCC are used for AFP assay via IMR. The results are shown with cross symbols (Figure 5). It was found that the AFP concentrations,
$\phi_{\mathrm{AFP}}$, for these patients ranged from 19 to $100 \mathrm{ng} / \mathrm{mL}$. As the AFP concentrations in the serum samples of 17 normal people were detected via IMR, it was found that the AFP concentrations were well below $15 \mathrm{ng} / \mathrm{mL}$, as shown with dots (Figure 5). The preliminary results shown in Figure 5 evidence the promising applications of IMR in diagnosing hepatocellular carcinoma.

\section{Conclusion}

In summary, it has been demonstrated that IMR shows the merits of a low detection threshold and nonsignificant interference. The low detection threshold for assaying AFP is improved by 15 times by using IMR instead of ELISA. This ultra-low detection threshold definitely suppresses the false negative, which is usually found for ELISA, when assaying low-concentration AFP at early-stage HCC. It was proven that jaundice, hemolysis, or hypertriglyceridemia did not interfere with the detection of targeted molecules in blood by IMR. Furthermore, the clinic tests for ten samples of human blood using IMR evidence the high sensitivity and high specificity for assaying AFP with IMR. These results reveal the high feasibility for assaying AFP in blood with IMR.

\section{Acknowledgments}

This work was supported by the National Science Council of Taiwan under grant numbers 98-2112-M-003-003, 98-2323B-003-001-CC2, NSC98-02752-M-002-016-PAE, and NSC 100-2120-M-002-015, the Department of Health under grant number DOH99-TD-N-111-008 and DOH100-TD-N111-008, and the Ministry of Economic Affairs of Taiwan under grant numbers 1Z970688, 1Z0990415 (SBIR), and S09800226-203 (JAID).

\section{Disclosure}

The authors report no conflicts of interest in this work.

\section{References}

1. Allan JC, Mencos F, Garcia-Noval J, et al. Dipstick dot ELISA for the detection of Taenia coproantigens in humans. Parasitol. 1993;107:79-85.

2. Mize TH, Taban I, Duursma M, et al. A modular data and control system to improve sensitivity, selectivity, speed of analysis, ease of use, and transient duration in an external source FTICR-MS. Intl J Mass Spect. 2004;235(3):243-253.

3. Li M, AlnoutiY, Leverence R, et al. Increase of the LC-MS/MS sensitivity and detection limits using on-line sample preparation with large volume plasma injection. J Chrom B. 2005;825(2):152-160.

4. Wu CM, Jian ZC, Joe SF, Chang LB. High-sensitivity sensor based on surface plasmon resonance and heterodyne interferometry. Sens Actu B Chem. 2003;92(1-2):133-136.

5. Hong CY, Wu CC, Chiu YC, et al. Magnetic susceptibility reduction method for magnetically labeled immunoassay. Appl Phys Lett. 2006;88(21):212512-1-3. 
6. Rothacker J, Ramsay RG, Ciznadija D, et al. A novel magnetic bead-based assay with high sensitivity and selectivity for analysis of telomerase in exfoliated cells from patients with bladder and colon cancer. Electrophoresis. 2007;28(23):4435-4446.

7. Silva EC, Gusmão LAP, Barbosa $\mathrm{CRH}$, et al. High sensitivity giant magnetoimpedance (GMI) magnetic transducer: magnitude versus phase sensing. Meas Sci Technol. 2011;22(3):35204-35208.

8. Hong CY, Chen WH, Jian ZF, et al. Wash-free immunomagnetic detection for serum through magnetic susceptibility reduction. Appl Phys Lett. 2007;90(7):74105-1-3.

9. Yang SY, Jian ZF, Chieh JJ, et al. Wash-free, antibody-assisted magnetoreduction assays on orchid viruses. J Virol Methods. 2008; 149(2):334-337.
10. Yang SY, Chieh JJ, Wang WC, et al. Magnetic nanoparticles for highsensitivity detection on nucleic acids via superconducting-quantuminterference-device-based immunomagnetic reduction assay. J Magn Magn Mater. 2011;323(6):681-685.

11. Yang SY, Jian ZF, Horng HE, et al. Dual immobilization and magnetic manipulation of magnetic nanoparticles. J Magn Magn Mater. 2008;320(21):2688-2691

12. Yang SY, Wang WC, Lan CB, et al. Magnetically enhanced high-specificity virus detection using bio-activated magnetic nanoparticles with antibodies as labeling markers. J Virol Methods. 2010;164(1-2):14-18.

\section{Publish your work in this journal}

The International Journal of Nanomedicine is an international, peerreviewed journal focusing on the application of nanotechnology in diagnostics, therapeutics, and drug delivery systems throughout the biomedical field. This journal is indexed on PubMed Central, MedLine, CAS, SciSearch ${ }^{\circledR}$, Current Contents ${ }^{\circledR} /$ Clinical Medicine,
Journal Citation Reports/Science Edition, EMBase, Scopus and the Elsevier Bibliographic databases. The manuscript management system is completely online and includes a very quick and fair peer-review system, which is all easy to use. Visit http://www.dovepress.com/ testimonials.php to read real quotes from published authors. 